\title{
Quantitative posture analysis of 2D, 3D, and optical microscope visualization methods for microsurgery tasks
}

\author{
Yu, Denny ${ }^{\mathrm{a}}$, Michael Sackllah ${ }^{\mathrm{a}}$, Charles Woolley ${ }^{\mathrm{a}}$, Steven Kasten ${ }^{\mathrm{b}}$, and Thomas Armstrong ${ }^{\mathrm{a}}$ \\ ${ }^{a}$ Center for Ergonomics, University of Michigan, 1205 Beal Ave., Ann Arbor, MI, USA, \\ ${ }^{b}$ Department of Surgery, University of Michigan, Ann Arbor, MI, 48109, USA,
}

\begin{abstract}
The purpose of this paper is to present a quantitative posture analysis of microsurgery tasks performed with different visualization methods. Microsurgery is traditionally performed using a binocular microscope; however surgeons are constrained by the optical eyepieces and are forced to assume joint angles that deviate away from neutral postures. This may be especially problematic for the neck and can increase surgeon discomfort and fatigue. Alternative visualization methods may improve surgeon posture by eliminating the constraints imposed by the microscope. This study examines both 2D and 3D heads-up displays as possible alternatives. Six subjects performed microsurgical tasks with each visualization methods for four hours. Quantitative posture analysis was done using Maxtraq software that tracks reflective markers on the subjects. The initial analysis of neck, upper arm, and elbow angles found significant differences between each display. A biomechanical analysis found that the differences in angles can result in loads on the neck joint that are twice as high in the microscope than the headsup displays. Although the alternative displays can result in better postures, improvements the display technology is needed to improve microsurgical task performance.
\end{abstract}

Keywords: microscope, heads-up display, posture analysis, surgery

\section{Introduction}

The purpose of this research is to quantify postural constraints imposed by different visualization equipment on subjects performing microvascular surgical technique skills tests. It is hypothesized that the utilization of microscope optical eyepieces imposes greater postural constraints than the use of a heads-up video display.

Musculoskeletal (MS) pain, fatigue, and discomfort affect both the comfort of microvascular surgeons and their ability to complete necessary tasks. Additionally, biomechanical and physiological factors may also affect their career longevity and how long they continue to practice.

Surgical jobs contain many ergonomic risk factors for MS symptoms because they require prolonged static posture of the head, neck, back, and upper extremities; however minimally invasive surgeries and microsurgeries present additional unique challenges to surgeons as a result of posture constraining equipment.

Posture quantification can be helpful in investigating how surgical equipment can lead to potential MS stress and strain. From biomechanical analysis, postures that deviate from neutral directly increase the biomechanical load on subject's joints. In addition to loads on the joint, the resulting moments also increase the EMG activity of subject's muscle [2]. Thus studying how surgeon posture changes with different equipment can create models important to preserving surgeon health.

Studies on laparoscopic surgeries have reported that restricted posture may induce fatigue by limiting natural changes in body postures [1]. It was also reported that $84 \%$ of laparoscopic surgeons use posture changes to minimize discomfort [4]. A major difference between laparoscopic surgery and microsurgery is the use of a microscope. *Microscope equipment is

\footnotetext{
* Corresponding author. Denny.z.yu@gmail.com
} 
essential to microsurgery but it limits the user's ability to change posture because head movement will cause surgeons to lose sight of the work site. The additional postural constraint may cause significant fatigue and discomfort to be experienced by microsurgeons.

Adjustment to the current microscope visualization methods can reduce fatigue and discomfort in microsurgeons. Video displays that stream magnified video into a flat-screen display may reduce fatigue by allowing surgeons greater degrees of freedom [3]. With the growing accessibility to 3-dimensional (3D) television displays, real-time video streams can even retain the stereoscopic information provided by traditional microscopes.

The long term aim is to model equipment and methods that minimize MS stress and strain specifically concerned with surgical tasks that require optical magnification. This study investigates the effects of different visualization methods (microscope, stereoscopic flat-panel display, and 2-dimensional flatpanel) on subject posture. Visual displays vary in degree of freedom allowed and the type of information presented to the user. Because the microscope is a significant posture constraint, an investigation of different displays and its relationship to MS discomfort could provide important groundwork for future interventions.

\section{Methods}

A laboratory study was conducted using a full factorial design where each subject was exposed to every treatment and every treatment order. Six participants with no surgical experience ( 3 males, 3 females) ages $21-26$ years-old with heights of $172 \pm 4 \mathrm{~cm}$ were recruited. Study was approved by University of Michigan Institutional Review Board.

Three visualization methods were tested; traditional optical microscopes, 2D video display monitor, and $3 \mathrm{D}$ video display monitor. For the optical microscope visualization method, a stereo dissection microscope (Scienscope Model EZ) was used. For the video display visualization methods, two analogue video eyepiece cameras were mounted onto the microscope and transmitted real-time images (less than $100 \mathrm{~ms}$ lag) to a Samsung 40" HDTV. For 2D visualization the signal from one of the two cameras was displayed and for 3D visualization signals from both cameras were encoded using the frame sequential 3D method and displayed on the monitor. Samsung wireless shutter glasses were used to view the $3 \mathrm{D}$ video. Data collection equipment included four subject observation video cameras and a camera inserted in the microscope. Subjects wore reflective markers on their head, arms, and hip that were later used in motion tracking as seen in Figure 1.

During testing the subjects performed four tasks for five minutes each for each visualization method. Three tasks were adopted for microsurgery from laparoscopic skills test and included Cup Drop Drill, Peg Board Transfer, and Circle Cutting tasks. Following recommendations from microsurgeons, a Millimeter Cutting task was also included. After performing all four tasks for one visualization method, the subjects took a 5 minute break during which they completed a discomfort survey. After the break, they repeated the process for the other two visualization methods. After using all three visualization methods, subjects took a 10 minute break and the whole procedure was conducted 2 more times using different orders of treatments.

Quantitative posture data was analyzed using Maxtraq motion tracking software (Innovision Systems Inc). Reflective markers on the subject where used to create angles as shown in Figure 1. Statistical analyses were conducted in accordance with Minitab's General Linear Model function.

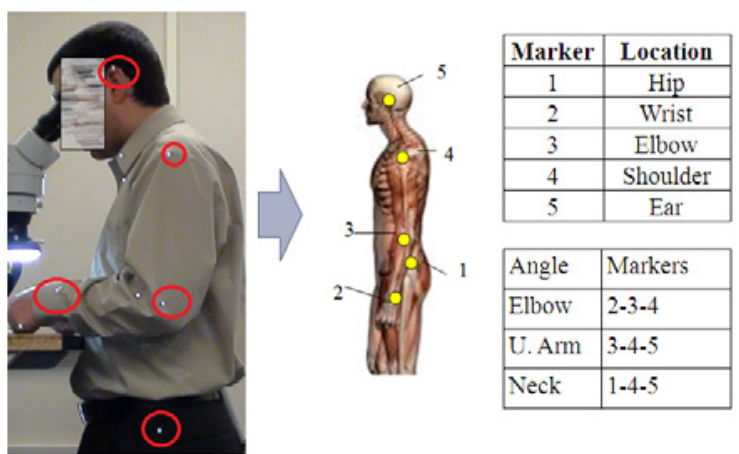

Fig 1: Marker location and conversion to angles

\section{Results}

\subsection{Objective Posture Analysis}

Posture angles were calculated from the subject markers. Five tracking markers were affixed to each subject to quantify elbow, upper arm, and neck flex- 
ion values in the two-dimension saggital plane. Example postures are shown in Figure 2.
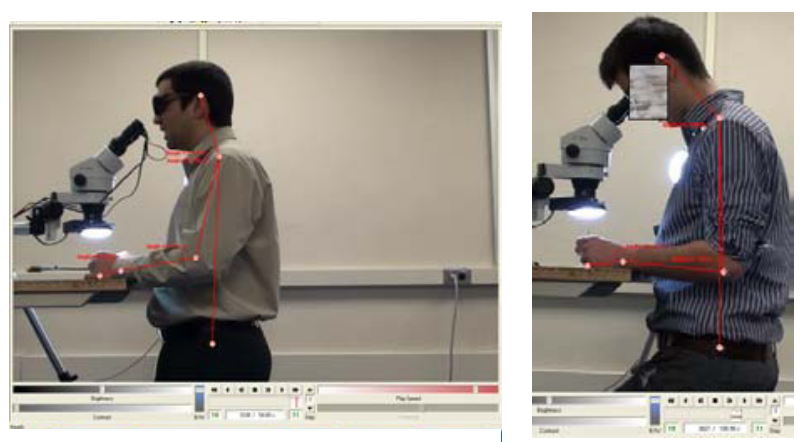

Fig 2: Examples of posture during experiment

Figure 3 illustrates a representative sample of the neck deviation for a subject while performing the Cup Drop Drill on each of the visualization methods. The sampling frequency was $10 \mathrm{~Hz}$. During the microscope trial, the subject sustained a static posture for approximately 60 seconds before he abruptly extended his neck. During the 2D trial, the subject adjusted his neck twice during the trial, at about 80 and 140 seconds. A sudden neck flexion is not evident in the $3 \mathrm{D}$ trial.

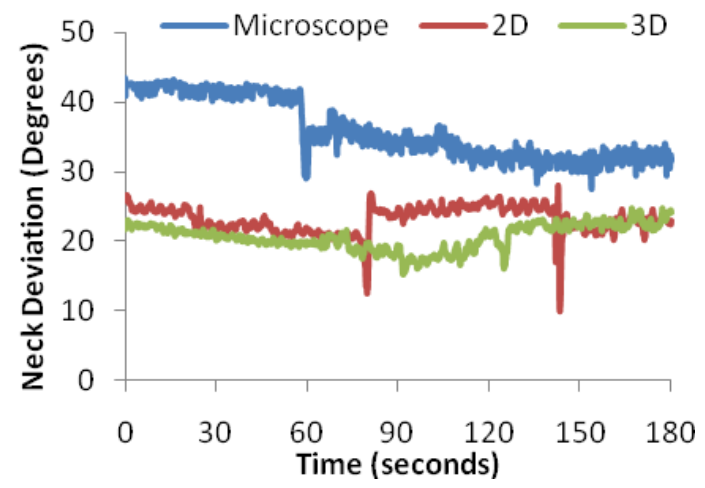

Figure 3: Neck deviation angle vs. time stratified by visualization method for Subject 6's Cup Drop Drill

Figure 4 depicts the percentage of time all the subjects were at a specific neck deviation throughout the trials. All the histograms appear relatively bellshaped around the mean deviation. In the $2 \mathrm{D}$ visualization method, the amount of time at each angle had a wider distribution than the microscope and $3 \mathrm{D}$ visualization method.
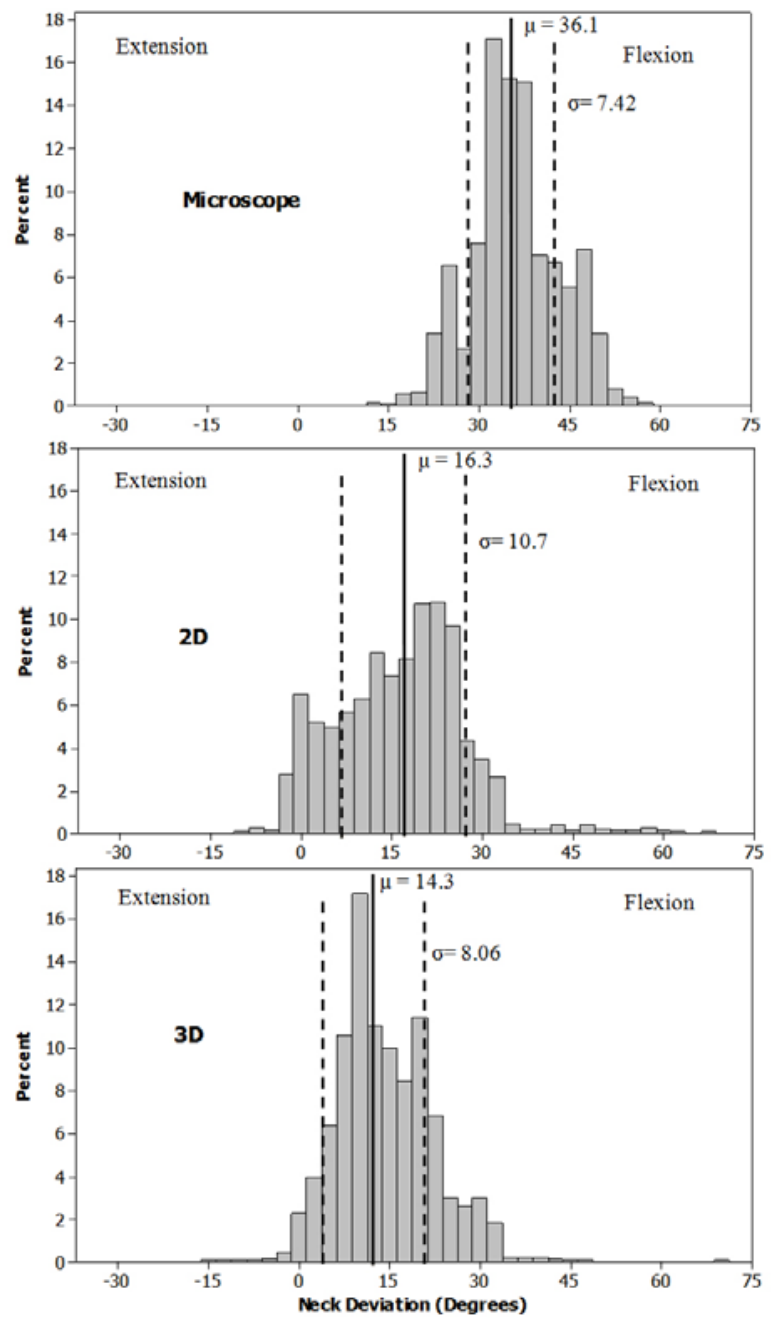

Fig 4: Neck deviation histogram for all subjects stratified by visualization method (top $=$ microscope, middle $=2 \mathrm{D}$, bottom $=3 \mathrm{D}$ ). Note that axes are identical scale.

The values in Table 1 and Figure 4 represent the average angle deviation from the neutral posture. Positive values indicate flexion and negative values indicated extension. Neutral positions were defined as $90^{\circ}$ flexion for the elbow and $0^{\circ}$ flexion for the upper arm and neck. Significant differences $(\mathrm{p}<0.05)$ were found between all visualization methods for elbow and neck flexion. For upper arm flexion, only the $3 \mathrm{D}$ visualization method yielded significantly smaller joint angle deviations. 
Table 1: Average and Standard Deviation of Posture Angles from all subjects

\begin{tabular}{|llll|}
\hline & Microscope & $2 \mathrm{D}$ & $3 \mathrm{D}$ \\
\hline Elbow & $-0.9^{\circ} \pm 10.1^{0 *}$ & $-14.4^{\circ} \pm 19.1^{0 *}$ & $-9.3^{\circ} \pm 10.9^{\circ *}$ \\
\hline $\begin{array}{l}\text { Upper } \\
\text { Arm }\end{array}$ & $12.0^{\circ} \pm 7.65^{\circ}$ & $11.9^{\circ} \pm 6.24^{\circ}$ & $10.1^{\circ} \pm 4.73^{\circ *}$ \\
\hline Neck & $36.1^{\circ} \pm 7.42^{\circ *}$ & $16.3^{\circ} \pm 10.7^{0 *}$ & $14.3^{\circ} \pm 8.06^{0 *}$ \\
\hline
\end{tabular}

\section{Discussion}

Quantitative postural analyses revealed that the optical microscope visualization method constrained neck and upper arm angles that deviated from neutral significantly more than both the display monitor visualization methods. The mean neck deviation for the microscope $\left(36.1^{\circ}\right)$ was more than 2.2 times greater than the other visualization methods $\left(16.3^{\circ}\right)$. This has important biomechanical impact on the MS discomfort in subjects. Assuming fixed length, $r$, of the neck-head length and fixed head weight, mg, the moment, $\mathrm{M}$, produced by gravity on the neck can be computed for a given neck flexion angle, $\theta$, as, $\mathrm{M}=$ $\mathrm{r}^{*} \mathrm{mg} * \sin (\theta)$. A $20^{\circ}$ increase in deviation angle increases the neck moment by a factor of 2.1 $\left(\sin \left(36.1^{\circ}\right) / \sin \left(16.3^{\circ}\right)=2.1\right)$.

In comparison with the other visualization methods, the 3D display resulted in upper arm and neck angles closer to neutral postures. Better neck angles may be creating a more relaxed upper body posture and reduce posture constraints at other area.

Although the $3 \mathrm{D}$ visualization method produced the best overall posture for all analyzed joints, a performance analysis indicated that the microscope outperforms the other visualization methods in every performance metric. This may be because of fami- liarity with the microscope or problems focusing the $3 \mathrm{D}$ visualization method.

Due to limitations in the posture tracking, only flexion angles in the two-dimension saggital plane were measured. Mild abduction of the elbow and supination of the arms were observed; however, this was not quantified in this study. Future studies should utilize a three-dimension tracking system to account for increased ranges of motion. A follow-up study should also be performed with increase sample size and on surgeon subjects to validate the findings. It is possible that additional training or better video technology might improve performance in the 3D and $2 \mathrm{D}$ displays.

\section{Acknowledgement}

The authors would like to acknowledge University of Michigan Center for Occupational Health and Safety Engineering NIOSH pilot grants for funding this research. Special thanks to Eyvind Claxton, Professor Jake Seagull, and Cooper Greene for their contributions and support on this study.

\section{References}

[1] Berguer, R., G. T. Rab, et al. (1997). "A comparison of surgeons' posture during laparoscopic and open surgical procedures." Surgical endoscopy 11(2): 139-142.

[2] Harms-Ringdahl, K., J. Ekholm, et al. (1986). "Load moments and myoelectric activity when the cervical spine is held in full flexion and extension." Ergonomics 29(12): 1539.

[3] E. Kantor, G. Berci, E. Partlow, M. Paz-Partlow (1991). A completely new approach to microlaryngeal surgery, The Laryngoscope, 101: 676-679, 1991.

[4] Park, A., G. Lee, et al. (2010). "Patients benefit while surgeons suffer: an impending epidemic." Journal of the American College of Surgeons 210(3): 306-313. 